\title{
Advances of Relativity Theory
}

\author{
Amrit S. Šorli ${ }^{1,2}$ \\ ${ }^{1}$ Bijective Physics Institute, Slovenia \\ ${ }^{2}$ Scientific research centre Bistra, Slovenija \\ sorli.bijective.physics@gmail.com \\ https://orcid.org/0000-0001-6711-4844 \\ Štefan Čelan \\ Scientific research centre Bistra, Slovenija \\ stefan.celan@bistra.si \\ https://orcid.org/0000-0003-3646-1469
}

\begin{abstract}
Advances of Relativity Theory are in the replacement of the space-time model with timeinvariant universal space that has a variable energy density. Every physical object with mass $\mathrm{m}$ and energy $\mathrm{E}$ is diminishing the energy density of space exactly for the amount of its energy. Lorentz factor has its origin in the variable energy density of universal space, we call it "superfluid quantum space" - SQS that is the primordial energy of the universe. Universal SQS is the absolute frame of reference for all observers as confirmed experimentally by the GPS system, which demonstrates that the relative rate of clocks is valid for all observers. A planet's perihelion precession and the Sagnac effect are the results of the SQS dragging effect.
\end{abstract}

Keywords: bijective research methodology, superfluid quantum space, Lorentz factor, planet's precession, Sagnac effect, GPS, dragging effect

PACS: 04.00

\section{Introduction}

In Special Relativity, time $t$ is the duration of photon motion along the $4^{\text {th }}$ coordinate: $X_{4}=i c t$. This confirms that $4^{\text {th }}$ coordinate is not time, $4^{\text {th }}$ coordinate too is spatial distance. Duration $t$ multiplied by light speed $c$ is spatial distance $X_{4}$. Minkowski manifold $X_{1}, X_{2}, X_{3}, X_{4}$ has four spatial coordinates and thinking that time is its $4^{\text {th }}$ coordinate is an utter mistake. Experimental data confirm that time $t$ is a duration of motion in universal space that is time-invariant in the sense that time is not its $4^{\text {th }}$ dimension. Development of Relativity Theory is based on three significant scientific discoveries: 
- space-time is not a fundamental arena of the universe; time is not the 4th dimension of space. Time is merely the duration of motion in timeinvariant universal space [1].

- Entanglement happens in time-invariant space only and not in time. Timeinvariant universal space is the immediate medium of quantum entanglement [2].

- Universal space is not "empty", space is the fundamental energy of the universe, in today physics called "superfluid quantum space" [3]. We will call it in this article time-invariant superfluid quantum space-SQS.

GPS proves that the relative rate of clocks on satellites relative to the Earth's surface is valid for all observers, including aeroplanes, trains, ships, and cars [4, 5]. This experimental fact, along with everyday experience, suggests a revision to our understanding of the famous Gedankenexperiment of one observer at a train station and another observer on a passing train. Standard physics textbooks describe that a clock at the station runs faster for the observer on the train, and the clock on the train runs slower for the observer at the station. In classic relativity both observers have their own 'internal time' inside the reference system in which they exist and both have an 'external time' that exists in the other observer reference system. This interpretation features four distinct times: the proper time of the observer at the station, the proper time of the observer in the train, the external time of the observer at the station, and the external time of the observer on the train. On the other hand, GPS proves that the relative velocities of clocks at the station and on the train are equally related to the rate of clocks on orbiting satellites, so are valid for both observers. If this were not so, then GPS could not work properly.

\section{Replacement of curvature of space with variable energy density of SQS}

Einstein tensor has three elements, curvature tensor on the left, Einstein constant and stress-energy tensor on the right side of the equation:

$$
G_{\mu \nu}=\kappa T_{\mu \nu}
$$

Curvature tensor $G_{\mu \nu}$ describes curvature of space due to the presence of a given mass that is expressed by the stress-energy tensor $T_{\mu \nu}$. Curvature tensor is useful only on the macro scale, 
it cannot be applied on microscale, for example proton. In this article curvature tensor will be replaced by the minimal energy density of SQS in the centre of the given physical object with the rest mass $m_{0}$. This formula is valid from the proton to the SMBH. Every physical object with energy $E$ and mass $m$ is diminishing energy density of SQS in its centre exactly for the amount of its energy and correspondent mass:

$$
E=m c^{2}=\left(\rho_{\text {Emax }}-\rho_{E \min }\right) \cdot V \quad \text { (2) [6], }
$$

where $\rho_{\text {Emax }}$ is density of SQS in interstellar space, $\rho_{\text {Emin }}$ is density of SQS in the centre of a given physical object and $V$ is the volume of physical object.

We can calculate the density of SQS at a given point on the distance $R$ from the centre of a given stellar object as follows:

$$
\rho_{R}=\rho_{\max }-\frac{3 m}{4 \pi(r+R)^{3}} \quad \text { (3) [6], }
$$

where $m$ is mass of the stellar object, $r$ is radius of the stellar object and $R$ is the distance from the centre of the stellar object to the point where we calculate $\rho_{R}$ density of SQS (Figure 1).

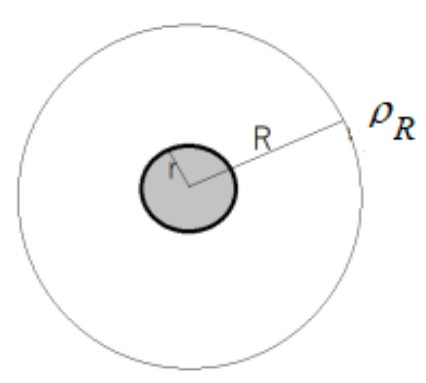

Figure 1: Energy density of SQS at the distance $R$ from the centre.

In Advanced Relativity curvature of space is replaced with variable energy density of space. More space is curved in GR, less is its energy density in Advanced Relativity.

\section{The Lorentz factor and variable energy density of the SQS}

Lorentz factor $\gamma$ expresses a diminished rate of clocks and a diminished velocity of material changes due to the motion. In the famous example of a train passing a station, $t^{\prime}$ is the elapsed time on the train and $t$ is the elapsed time at the station, such that

$$
t^{\prime}=\gamma \cdot t
$$


This diminished rate of clocks on the train has its origin in the decreased energy density of the SQS inside the train. In general, a moving system interacts with the SQS energy so that the higher the velocity $\mathcal{V}$, the stronger the interaction and more SQS energy is integrated into the moving object, which in turn increases its mass $m$ according to:

$$
m=\gamma \cdot m_{0}=m_{0}+\frac{E K}{c^{2}}
$$

where $m_{0}$ is the object's rest mass, EK is moving object kinetic energy in the form of integrated energy of SQS and $\gamma$ is the Lorentz factor.

Out of equation (2) follows, the formula for the minimal energy density of SQS $\rho_{E m i n}$ in the rest train is following:

$$
\rho_{\text {Emin }}=\rho_{\text {Emax }}-\frac{m_{0} \cdot c^{2}}{V}
$$

Formula for the $\rho_{\text {Emin }}$ for the moving train is following:

$$
\rho_{\text {Emin }}=\rho_{\text {Emax }}-\gamma \cdot \frac{m_{0} \cdot c^{2}}{V}
$$

This decreased energy density of the SQS $\rho_{\text {Emin }}$ causes the rate of the clock on the train to run slower. According to Equation (7), we can express the Lorentz factor as follows:

$$
\gamma=\frac{\left(\rho_{E \max }-\rho_{E \min }\right) \cdot V}{m_{0} \cdot c^{2}}
$$

By replacing rest mass $m_{0}$ with relativistic mass $m$ we get:

$$
\gamma=\frac{\left(\rho_{E \max }-\rho_{E \min R}\right) \cdot V}{m \cdot c^{2}}
$$

where $\rho_{E \operatorname{minR}}$ is the additionally diminished energy density in the centre of the train because moving train matter is absorbing some of the SQS energy which increases train's relativistic mass. Formula (9) confirms Lorentz factor depends on the relativistic mass $m$, it means it depends on the speed $v$ of the physical object. So, the higher is the speed $v$, the stronger is the 
interaction of the object with the SQS, absorption of the SQS energy is greater, and the density of the SQS in the centre of the moving object becomes smaller. With a smaller density of the SQS in the centre of the train (and any other stellar object), the rate of the clock is slower:

increased velocity $\rightarrow$ increased absorption of the SQS energy $\rightarrow$ decreased energy density of the SQS $\rightarrow$ decreased rate of a clock.

We conclude that the relative rate of clocks and the relative velocity of material changes depend only on the variable energy density of the SQS. For example, muon decay when approaching the Earth's surface decreases because their velocity increases [7]. With this increasing velocity, the minimal energy density of the SQS of the muon decreases, and the time of decay increases. The duration of this decay does not depend on the selected reference system nor the chosen observer. A muon's relativistic decay is valid for all observers and is determined only by the variable energy density of the SQS.

\section{Advances of Special Relativity}

The Lorentz factor is not related to some inertial systems as it depends only on the variable energy density of the SQS. From this perspective, the SQS is the absolute reference system in which elementary particles, physical objects, and stellar objects move. Einstein's idea that there is no absolute motion is again examined here. Because of the abolishment of the notion of an ether at the end of the $19^{\text {th }}$ century, the idea of absolute motion was also abandoned. Introduction of a SQS as the fundamental arena of the universe allows the reintroduction of absolute motion, which is defined as the motion of a physical object with a SQS as the absolute reference frame.

After ether was taken out of the physics, Einstein introduced the concept of 'relative motion', which means there is no absolute reference system in which any given motion occurs. A moving object's velocity can be measured only in comparison with another object's velocity. In the model presented in this article, the speed of light is constant for all observers because all observers exist in the same SQS and light is the vibration of the SQS. SQS in the universe is not 'empty', SQS is the fundamental energy of the universe. Such understanding offers a good example of relative and absolute motions. In physical SQS which is the absolute frame of reference we distinguish relative and absolute velocity. You imagine you drive a car with a speed of $110 \mathrm{~km}$ per hour. 500 metres in front of you is a car moving with a speed of $120 \mathrm{~km}$ per hour. An accident happens and the car in front of you crashes in the wall along the street. 
The driver luckily survives but the car is totally destroyed. This simple example proves that the 'relative speed' of $10 \mathrm{~km}$ per hour of the car in front of you was not 'real.' The real velocity of the car is the velocity regarding the highway, which is $120 \mathrm{~km}$ per hour. The same is valid for light speed, whose 'real' velocity is the velocity of the light in the physical SQS and is valid for all observers.

By assuming the SQS as the absolute reference frame, we can develop an SR theory without contradictions as those that exist with the current SR in the thought experiment of twophoton clocks. Here, we place two identical photon clocks on a moving train where one is positioned horizontally in the direction of motion, and the other is positioned vertically. According to the idea of "length contraction," the horizontal photon clock will shorten in length and tick faster compared to the vertically oriented clock that will not diminish in length. This scenario leads to a contradiction as SR does not predict that the two clocks in the same inertial system will have different rates. The solution is available through the development of an SR model in a three-dimensional Euclidean SQS with a Galilean transformation and Selleri formula for the variable rate of clocks with no occurrence of "length contraction" [8]:

$$
t^{\prime}=\sqrt{1-\frac{v^{2}}{c^{2}}}
$$

there $t^{\prime}$ is elapsed time in thew moving train and $t$ is elapsed time on the train-station. SR equipped with this formalism also describes successfully all phenomena previously described by classical SR [9].

A second contradiction occurs with the rate of the vertical photon clock on the moving train from the perspective of the observer at the station. The classical interpretation states that for the observer at the station, the vertical photon clock ticks slower because they see the photon in the clock moves in a 'zig-zag' direction [10], as illustrated in Figure 1. 


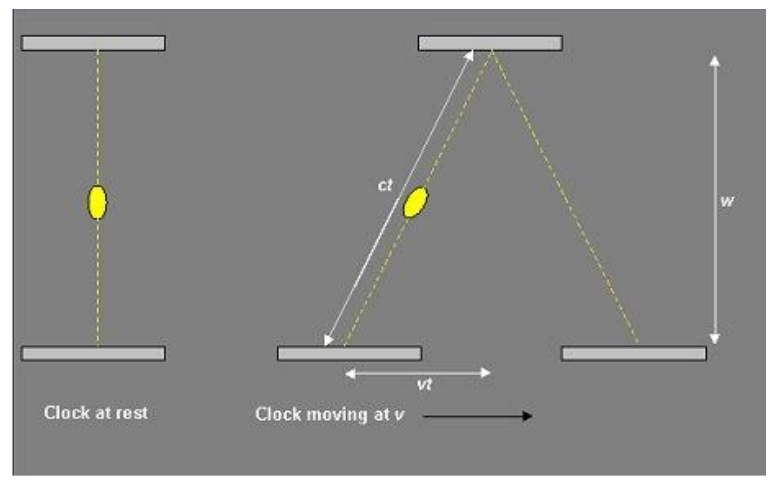

Figure 1. An observer at rest, seeing a moving clock photon.

This explanation may appear illogical because the optical illusion of the stationary observer cannot slow the rate of the moving clock. Instead, in the moving train, the density of the SQS diminishes, causing a reduced velocity of the photon. With the diminishing of SQS energy density also the velocity of light diminishes a bit. Therefore, the moving vertical photon clock ticks slower and its rate is valid for all observers.

\section{Advances of General Relativity}

In the model presented in this paper, the rotation of stellar objects also causes rotation of the surrounding SQS. For example, the rotation of the SQS around the Sun causes precession of the planets [11] according to

$$
\sigma=\frac{24 \pi^{3} L^{2}}{T c^{2}\left(1-e^{2}\right)}
$$

where the perihelion shift $\sigma$ is expressed in radians per revolution, $L$ is the semi-major axis, $T$ is the orbital period, $c$ is the speed of light, and $e$ is the orbital eccentricity. The mass of the Sun is not included as there is also no mass of a planet, so these masses do not affect the precession of the planets. In the model of SQS, the perihelion shift $\sigma$ depends on the rotation of the SQS caused by the rotation of the Sun, which in turn pushing the planets and causes a perihelion precession. With increasing distance from the Sun, the impact of the rotating SQS on planets diminishes along with the precession of the perihelion.

Irregular and spiral galaxies comprise approximately $60 \%$ of all galaxies in the universe. In the centre of most spiral galaxies exist a rotating black hole [12], which also rotates the 
surrounding SQS causing the shape of the spiral galaxy. Dragging effects between the rotating black hole and rotating SQS diminishes with the distance from the black hole leading to the spiral geometry.

In the central black hole, matter is continuously disintegrating back into the energy of the SQS providing a form of rejuvenation of the universe where "old matter" is transformed back into the energy of the SQS [6].

"The rotating space around the black hole as the physical origin of the surrounding rotating disc was discovered recently, "as if black holes weren't mysterious enough, astronomers using NASA's Hubble SQS Telescope have found an unexpected thin disk of material furiously whirling around a supermassive black hole at the heart of the magnificent spiral galaxy NGC 3147, located 130 million light-years away. The conundrum is that the disk shouldn't be there, based on current astronomical theories" [13]. In current astronomical theories, stellar objects rotate in an "empty space." However, in model presented in this article, stellar objects rotate in the medium of a quantum SQS, and this rotation, in turn, rotates the SQS. Therefore, the rotating SQS builds the rotating disc of the spiral galaxy NGC 3147.

In GPS, Sagnac effect corrections make the system work [14]. Essentially, a signal when moving from A to B in the direction of Earth's rotation needs less time compared to when moving from $\mathrm{B}$ to $\mathrm{A}$ in the direction opposing Earth's rotation. In a quantum SQS model presented in this article, light has a constant speed regardless of the quantum SQS's motion. So, when moving from A to B, light needs less time because it is moving in the same direction as the quantum SQS.

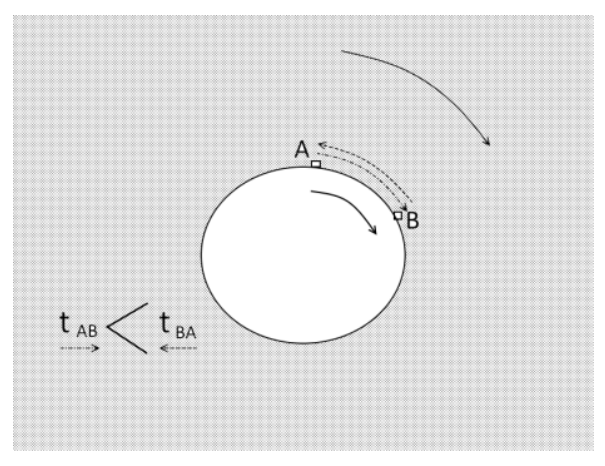

Figure 2. A light signal's time dilation due to the rotation of the quantum SQS.

The Michelson-Morley experiment demonstrated a null result because the area of the SQS around the Earth is not only rotating with the Earth but is also moving with the Earth, as shown in Figure 3. 


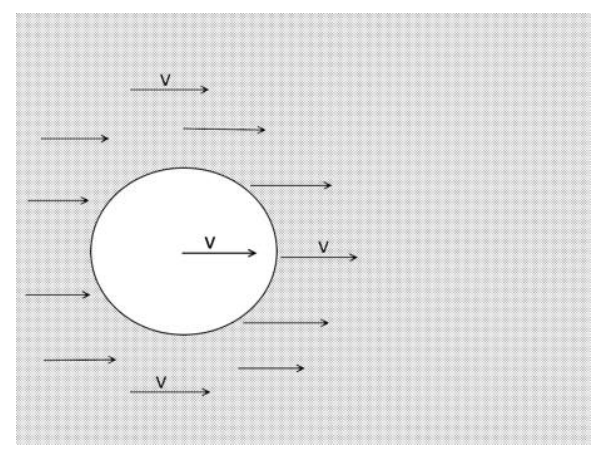

Figure 3. The SQS moves with the Earth.

So, the negative outcome of Michelson-Morley abolished the ether model. According to bijective research methodology, SQS is neither filled with ether, nor is it 'empty.' Instead, SQS contains material objects that contain energy. Energy and matter cannot exist in an 'empty' SQS deprived of all physical properties, so in this article universal SQS which is quantum (it interacts with physical objects) is understood as the primordial energy of the universe. A photon is a wave of the quantum SQS, and the velocity of this photon wave is the speed of light, $c$. The photon velocity $c$ is invariant with respect to the SQS's motion as it appears through the Sagnac effect. The photon velocity diminishes minimally when a photon moves through a stronger gravity where the density of the SQS is lower, as is the case with the Shapiro experiment, as will be described in Section 5.2.

Motion and rotation of the SQS with physical objects is referred to as the 'SQS dragging effect' in this article. This effect was measured by Josef Lense and Hans Thirring in 1918 and was called 'frame-dragging' due to space-time being distorted by rotating objects [15]. Recent research confirms that this 'space-time' model has no physical reality [6], so it cannot be dragged by rotating or moving objects. According to bijective research methodology, an adequate term would be the 'SQS dragging effect.'

In this article, the idea of "length contraction" and "time dilation" do not exist. Length contraction in SR is only a mathematical tool with no physical reality. By using "length contraction," Einstein achieved a constancy of light in all inertial systems. On the other hand, all observers measure the same value for the velocity of light because the light is the vibration of the medium (quantum SQS) in which all observers move. Also, time, being the fourth dimension of SQS, does not "dilate," and instead, is a result of the velocity of material changes (the rate of clocks included) that depends on the variable density of the SQS [6]. 


\subsection{Gravitational redshift}

Gravity has its origin in SQS fluctuations that interact with photons to diminish their frequency, which is referred to as 'gravitational redshift.' When light from distant galaxies reaches the Earth, its frequency is lower. On its path to Earth, light loses some of its energy because it is moving against the gravity flow of the SQS that points toward the direction of galaxies, so that

$$
E_{\text {photonEarth }}=E_{\text {photongalaxy }}-\Delta E
$$

where $E_{\text {photon.galaxy }}$ is the energy of the photon at the galaxy, $E_{\text {photon.Earth }}$ is the energy of the arrived photon at the Earth, and $\Delta E$ is the loss of energy due to the gravitational flow of the SQS,

$$
\Delta E=h \cdot \Delta v
$$

where $h$ is Planck's constant and $\Delta v$ is the decrease of the photon frequency due to gravitational SQS fluctuations.

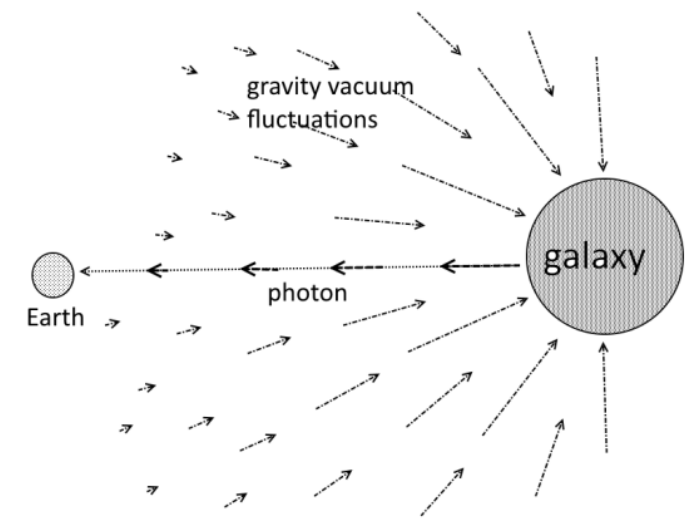

Figure 4. The redshift of light arriving from galaxies caused by SQS fluctuations.

Because of different densities of the SQS and corresponding SQS fluctuations that carry gravity, the frequency of light also changes when moving from the source to the receiver above the Earth's surface. In a Harvard University experiment, a source on the Earth's surface and a receiver at the height of 22,5 meters were positioned, as illustrated in Figure 5. 


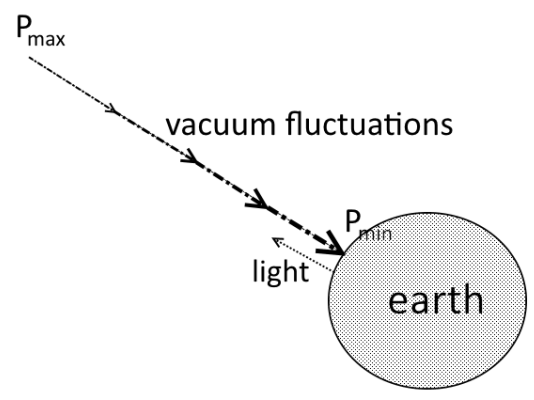

Figure 5. The redshift of light from the Earth's surface.

The Mössbauer effect was used to measure the difference between y-ray emission and absorption frequencies at each end of the experiment. The measurement accuracy was $\Delta \omega / \omega \approx 10^{-15}$, which shows a change of light frequency as

$$
\frac{\Delta \omega}{\omega}=\frac{G M}{R^{2} c^{2}} \cdot h
$$

where $M$ and $R$ are the mass and radius of the Earth, respectively [16].

We can substitute into Equation (14) for the mass $M$ with the $\frac{\left(\rho_{E \max }-\rho_{E \min }\right) \cdot V}{c^{2}}$ from Equation (3) as:

$$
\frac{\Delta \omega}{\omega}=\frac{G \cdot\left(\rho_{E \max }-\rho_{E \min }\right) \cdot V}{R^{2} \cdot c^{4}} \cdot \mathrm{h}
$$

which can be expressed as:

$$
\begin{aligned}
& \frac{\Delta \omega}{\omega}=\frac{G \cdot\left(\rho_{E \max }-\rho_{E \min }\right) \cdot 4 \pi R^{3}}{3 R^{2} \cdot c^{4}} \cdot \mathrm{h} \\
\frac{\Delta \omega}{\omega}= & \frac{4 \pi R \cdot G \cdot\left(\rho_{E \max }-\rho_{E \min }\right)}{3 c^{4}} \cdot \mathrm{h}
\end{aligned}
$$


Equation (16) confirms that gravitational redshift depends on the minimal energy density of the SQS $\rho_{E \min }$ in the Earth's centre. SQS fluctuation in the direction from $\rho_{\max }$ towards $\rho_{\min }$ are explaining so called "tired light" model of astronomer Fritz Zwicky. Zwicky proposed that light is losing some of the energy when travelling vast distances from the galaxies to the planet Earth [17].

In model of Relativity here presented SQS fluctuations from $\rho_{\max }$ towards $\rho_{\min }$ are causing the Pioneer anomaly which means the observed deviation from predicted accelerations of the Pioneer 10 and Pioneer 11 spacecraft after they passed on their trajectories out of the Solar System [18].

\subsection{Shapiro gravitational delay}

In 1964, Shapiro [19] measured the decreased velocity of light in a gravitational field, as observed by the speed of a light signal diminishing when passing the gravitational field of the Sun. Shapiro's result is understood by today's physics as a 'gravitational time delay' caused by spacetime dilation, which increases the path length. According to bijective research methodology, this interpretation appears not to be exact as Shapiro did not measure spacetime dilation. In SR, the element of 'spacetime dilation' has no bijective correspondence in the physical world as it has never been observed in physics that spacetime or space are dilating. According to bijective research methodology, Shapiro's result should be termed the 'gravitational diminishing of light-speed' caused by the diminished density of the SQS. In SQS with a given gravitational field, the density of the SQS is diminished that causes a minimal diminishing of the speed of light as defined by the permittivity and permeability of the SQS

$$
c=\frac{1}{\sqrt{\mu_{0} \varepsilon_{0}}}
$$

where $\mu_{0}$ is the magnetic permeability and $\varepsilon_{0}$ is electric permittivity of the SQS where there is no influence of gravity, and the density of the SQS is at its maximum $\rho_{\max }$. In the SQS with a gravity field, the density of the SQS decreases and causes minimal diminishing of the permittivity and permeability, which in turn result in the minimal diminishing of the speed of light, as presented by Masanori, "it is known that the speed of light depends on the gravitational potential. In the gravitational fields, the speed of light becomes slow, and time dilation occurs. 
In this discussion, the permittivity and permeability of free SQS are assumed to depend on gravity and are variable" [20]. Minimal variability of the speed of light caused by a gravity field maintains SR because its first postulate is valid only in SQS where gravity is absent. The electric permittivity in flat SQS with no gravity is $\varepsilon_{0}$, and magnetic permeability in flat with no gravity SQS is $\mu_{0}$. Following Puthoff, on the surface of stellar object, permittivity and permeability are

$$
\begin{aligned}
& \varepsilon=K \cdot \varepsilon_{0} \\
& \mu=K \cdot \mu_{0}
\end{aligned}
$$

where the SQS dielectric constant $K$ on the surface of a stellar object is

$$
K \approx 1+\frac{2 G M}{r c^{2}}
$$

with $G$ being the gravitational constant, $M$ the mass, and $r$ the distance from the origin located at the centre of the mass $M$ [16].

Combining equation (2) and (20) we can write following equation:

$$
K \approx 1+\frac{2 G \cdot\left(\rho_{\max }-\rho_{\min }\right) \cdot V}{r c^{4}}
$$

which shows the SQS dielectric constant depends on mass $m$ of the given stellar object and consequently on the variable energy density of SQS. In this sense, a diminished energy density of the SQS on the surface of a given stellar object increases permittivity and permeability of the SQS which, in turn, minimally decreases the velocity of light as:

$$
c=\frac{1}{\sqrt{\varepsilon \cdot \mu}}
$$


where $\varepsilon$ is the electric permittivity and $\mu$ is its magnetic permeability of the SQS where there is gravitational field. From this, it follows that the Shapiro gravitational time dilation has its origin in the diminished energy density of the SQS near the stellar objects, which increases the dielectric constant $K$ of the SQS and minimally decreases the velocity of light. In other words,

diminishing SQS energy density $\rightarrow$ increases the dielectric constant $\rightarrow$ increases the electric permittivity of the SQS $\rightarrow$ increases the magnetic permeability of the SQS $\rightarrow$ decreases the velocity of light

The classic textbook explanation of the Shapiro experiment is that in stronger gravity, time, as the fourth physical dimension of SQS, dilates causing light to need more time to reach the point $\mathrm{B}$ from point $\mathrm{A}$ in a SQS time that acts as the fundamental arena of the universe. This article shows that through a bijective interpretation of data, where data are not interpreted but read directly, requires an exact explanation where the velocity of light is minimally diminishing in a gravity field due to a diminished energy density of the SQS.

Doppler effect proves the second postulate of SR, which states that "the speed of light $c$ is a constant, independent of the relative motion of the source." The observer exists in SQS, and a photon is the vibration of the same SQS. When the observer moves toward or away from the source of light, they will experience the Doppler effect. With the understanding that the moving observer and the source both exist in the same SQS and that light is the vibration of the SQS, the second SR postulate becomes logical. The observer sees the light with a given frequency coming from the source. When the observer moves away from or closer to the source, the frequency of the light diminishes or increases, respectively. In the Doppler effect, the velocity of light $c$ for the moving observer remains constant because light is a wave of the SQS in which the observer moves and in which the source of light exists.

\subsection{Gravitational lens}

SQS fluctuations bend light, which we refer to as a 'gravitational lens,' and this bending of light as it passes the Sun is one proof of General Relativity. The SQS fluctuations near the Sun's surface are strongest and push the photons, causing them to bend, as illustrated in Figure 6. 


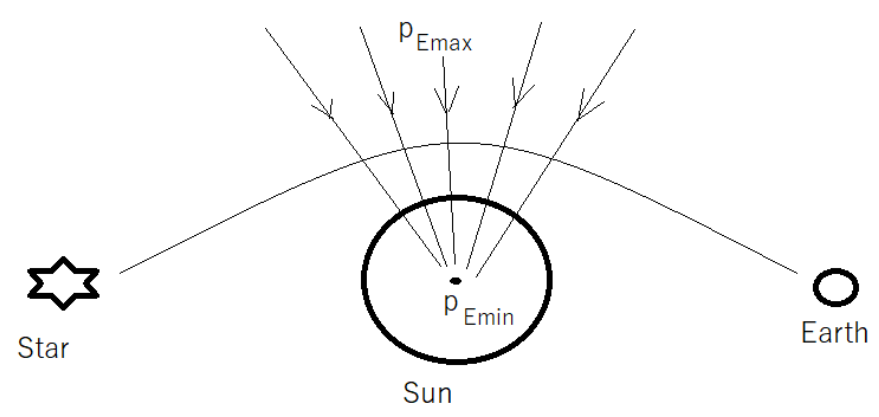

Figure 6. SQS fluctuations bending light around the Sun.

Gravity SQS fluctuations bend the photon's trajectory, which we call 'gravitational lens.' Einstein's formula for the bending of light as it passes the Sun [21] is expressed as:

$$
\delta=\frac{4 G \cdot M_{S}}{c^{2} \cdot b}
$$

where $\delta$ is the angle of deflection, $M_{S}$ is the mass of the Sun, $c$ is the speed of light, and $b$ is the minimum distance between the trajectory and the centre of the Sun. The mass of the Sun, $M_{S}$, can be expressed according to Equation (2), which we can combine with Equations (23) and to obtain

$$
\delta=\frac{4 G \cdot\left(\rho_{E \operatorname{man}}-\rho_{E \min }\right) \cdot V}{c^{4} \cdot b}
$$

Equation (24) confirms that SQS fluctuations that carry gravity are directed from SQS where the energy density of the SQS is $\rho_{E \max }$, and the density of the SQS is $\rho_{E \min }$ in the centre of the Sun, as in Figure 6. These SQS fluctuations push the photons, causing light deflection. Bijective research methodology confirms that light passing the Sun is not deflected as a result of the curvature of SQS. Measurements by NASA have proven that the universe's space has a Euclidean shape [22] so that the curvature of SQS in the GR model is merely a mathematical description of the variable density of the SQS. Light is deflected around gravitational objects, such as the Sun, due to a push from SQS fluctuations. 


\subsection{Structure of the black hole inside the horizon}

In the model presented in this article, in the centre of a black hole, the energy density of the SQS is so low that atoms become unstable and disintegrate back into the elementary particles [6]. In current models of the black holes, the energy density of the SQS is the missing element. "The recent observation by the Event Horizon Telescope of the shadow of the supermassive black hole M87 has provided the most direct image of a black hole thus far, opening the gate directly observe long-existing and weakly perturbed black holes 42,43 . Our findings indicate that gravitational waves will allow us to dig even deeper and resolve the evolving structure of the horizon itself of newborn and fully quantum al ones" [23]. In this article is considered that inside the black hole horizon in the centre of a black hole atoms become unstable and fall apart into elementary particles that forms huge jets of AGNs. These jets are "fresh energy" for the formation of new stars. Black holes are rejuvenating systems of the universe.

In this article gravitational waves are not represented as "ripples in the fabric of spacetime" [24] because space-time, as the fundamental arena of the universe, does not exist. Gravitational waves are ripples of the SQS. Gravitational waves change the permittivity and permeability of SQS. As gravitational waves enter the LIGO interferometer, they changed permeability and permittivity of the SQS which minimally changes the speed of light moving in the beams of the interferometer. This minimal change in the speed of light is what is directly measured by LIGO. No direct data exists to confirm that the length of the beams of the interferometer change due to the gravitational waves. How the subtle phenomena of a gravitational wave could shrink or elongate the length of the interferometer beams, which have a solid iron-concrete core, is an unanswered question. The model here presented solves this question through the reading of the available data as required by bijective research methodology. What is measured by LIGO is the minimal change in light speed due to minimal variations of the permittivity and permeability of the SQS caused by the gravitational wave entering the interferometer.

In the model presented, there exists the similarity between the photon and gravitational wave as both are a wave of the universal SQS. Recent research confirms gravitational waves have a speed close to the speed of the photon, "since the recent major discovery in physics, the first measurement of gravitational waves, achieved by the LIGO/Virgo collaboration, several events have been registered. In particular, the merging of two neutron stars detected with its electromagnetic counterpart by the FERMI satellite has led to implications of paramount importance. One of them is the speed of gravitational waves now constrained to be extremely 
close to that of light, at the 10-15 level, at low redshifts" [25]. In the model presented in this article the photon and gravitational wave are both waves of the SQS.

\section{Conclusions}

Development of Relativity Theory with bijective research methodology leads to the abolishment of space-time as the fundamental arena of the universe that is replaced with timeinvariant superfluid quantum space (SQS) as the fundamental energy of the universe where time is the duration of material change, i.e. motion. Universal SQS is quantum in the sense that has a variable energy density, it rotates and moves together with physical objects. Light is the excitation of SQS; its velocity is minimally diminished in regions where SQS has lower energy density. 'Relativistic phenomena' have a physical origin in the variable energy density of the SQS and in the SQS dragging effect.

\section{References:}

1. Fiscaletti D, Sorli A. Perspectives of the numerical order of material changes in timeless approaches in physics, Foundations of Physics, 45(2):105-133, (2015).

2. Fiscaletti D, Sorli A. Searching for an adequate relation between time and entanglement. Quantum Studies: Mathematics and Foundations 2017; 4(4):357-74.

3. Sorli A., Čelan Š., Superfluid Quantum Space as the Unified Field Theory, MDPI preprint https://www.preprints.org/manuscript/202011.0460/v1

4. Neil A., Relativity and GPS, Physics Today (2002).

5. Agnew, D.C., Larson, K.M., Finding the repeat times of the GPS constellation. GPS Solutions. 11 (1): 71-76. (2007).

6. Sorli A. S., Mass-Energy Equivalence Extension onto a Superfluid Quantum space, Scientific Reports, volume 9, Article number: 11737 (2019) https://www.nature.com/articles/s41598-019-48018-2

7. Liu L., The Speed and Lifetime of Cosmic Ray Muons, http://web.mit.edu/lululiu/Public/pixx/not-pixx/muons.pdf (2007.

8. Sorli, A., Klinar, D., Fiscaletti, D., New insights into the special theory of relativity, Phys. Essays 24,2 (2011).

9. Fiscaletti D., Sorli A., About a new suggested interpretation of special theory of relativity within a three-dimensional Euclid SQS. Annales Universitatis Mariae Curie-Sklodowska, sectio AAA-Physica, 68: 39-62. (2015).

10. Philip Harris, Special Relativity, pp. 29 https://web.stanford.edu/ oas/SI/SRGR/notes/srHarris.pdf

11. Einstein, Albert (25 de noviembre de 1915). «Die Feldgleichungun der Gravitation». Sitzungsberichte der Preussischen Akademie der Wissenschaften zu Berlin: 844-847 (1915).

12. Loveday J., The APM Bright Galaxy Catalogue, Monthly Notices of the Royal Astronomical Society. 278 (4): 1025-1048. arXiv:astro-ph/9603040 (1996). 
13. NASA, Hubble Uncovers Black Hole Disk that Shouldn't Exist, https://www.nasa.gov/feature/goddard/2019/hubble-uncovers-black-hole-thatshouldnt-exist (2019).

14. Ashby N., The Sagnac Effect in the Global Positioning System. In: Rizzi G., Ruggiero M.L. (eds) Relativity in Rotating Frames. Fundamental Theories of Physics, vol 135. Springer, Dordrecht (2004).

15. Lense, J., Thirring H., "Über den Einfluss der Eigenrotation der Zentralkörper auf die Bewegung der Planeten und Monde nach der Einsteinschen Gravitationstheorie". Physikalische Zeitschrift. 19: 156-163. [On the Influence of the Proper Rotation of Central Bodies on the Motions of Planets and Moons According to Einstein's Theory of Gravitation] (1918).

16. Puthoff H.E., Polarizable-SQS(PZ) presentation of general relativity, Found.Phys. 32, 927-943. (2002).

17. Zwicky F., On the Red Shift of Spectral Lines through Interstellar SQS. PNAS 15:773-779. (1929).

18. Nieto, M. M.; Turyshev, S. G.,Finding the Origin of the Pioneer Anomaly. Classical and Quantum Gravity. 21(17): 4005-4024. (2004).

19. Shapiro I.I., Fourth Test of General Relativity. Physical Review Letters. 13 (26): 789-791. (1964).

20. Masanori S., Gravitational effect on the refractive index: A hypothesis that the permittivity, $\varepsilon 0$, and permeability, $\mu 0$ are dragged and modified by the gravity https://arxiv.org/vc/arxiv/papers/0704/0704.1942v3.pdf

21. Albert Einstein, Lens-like action of the star by the derivation of light in the gravitational field, Science, Vol. 84, Issue 2188, pp. 506-507. (1936).

22. NASA https://wmap.gsfc.nasa.gov/universe/uni_shape.html (2014).

23. Juan Calderón Bustillo, Chris Evans, James A. Clark, Grace Kim, Pablo Laguna, Deirdre Shoemaker, Gravitational-wave imaging of black hole horizons: Post-merger chirps from binary black holes, https://arxiv.org/abs/1906.01153 (2019).

24. LIGO, Gravitational Waves: Ripples in the fabric of SQS-time, https://SQS.mit.edu/LIGO/more.html (2008).

25. Louis Perenon, Julien Bel, Roy Maartens, Alvaro de la Cruz-Dombriz, Optimising growth of structure constraints on modified gravity, https://arxiv.org/abs/1901.11063 (2019). 